\title{
THE DEPRESSION OF A GRAPH AND $k$-KERNELS
}

\author{
Mark Schurch and Christine Mynhardt \\ Department of Mathematics and Statistics \\ University of Victoria, P.O. Box 3060 STN CSC \\ Victoria, BC, Canada V8W $3 R 4$ \\ e-mail: mschurch@uvic.ca \\ mynhardt@math.uvic.ca
}

\begin{abstract}
An edge ordering of a graph $G$ is an injection $f: E(G) \rightarrow \mathbb{R}$, the set of real numbers. A path in $G$ for which the edge ordering $f$ increases along its edge sequence is called an $f$-ascent; an $f$-ascent is maximal if it is not contained in a longer $f$-ascent. The depression of $G$ is the smallest integer $k$ such that any edge ordering $f$ has a maximal $f$-ascent of length at most $k$. A $k$-kernel of a graph $G$ is a set of vertices $U \subseteq V(G)$ such that for any edge ordering $f$ of $G$ there exists a maximal $f$-ascent of length at most $k$ which neither starts nor ends in $U$. Identifying a $k$-kernel of a graph $G$ enables one to construct an infinite family of graphs from $G$ which have depression at most $k$. We discuss various results related to the concept of $k$-kernels, including an improved upper bound for the depression of trees.
\end{abstract}

Keywords: edge ordering of a graph, increasing path, monotone path, depression.

2010 Mathematics Subject Classification: 05C78, 05C38.

\section{REFERENCES}

[1] A. Bialostocki and Y. Roditty, A monotone path in an edge-ordered graph, Int. J. Math. Math. Sci. 10 (1987) 315-320. doi:10.1155/S0161171287000383

[2] A.P. Burger, E.J. Cockayne and C.M. Mynhardt, Altitude of small complete and complete bipartite graphs, Australas. J. Combin. 31 (2005) 167-177.

[3] A.R. Calderbank and F.R.K. Chung and D.G. Sturtevant, Increasing sequences with nonzero block sums and increasing paths in edge-ordered graphs, Discrete Math. 50 (1984) 15-28.

doi:10.1016/0012-365X(84)90031-1 
[4] G. Chartrand and L.M. Lesniak, Graphs and Digraphs (Third Ed.) (Wadsworth, 1996).

[5] V. Chvátal and J. Komlós, Some combinatorial theorems on monotonicity, Canad. Math. Bull. 14 (1971) 151-157. doi:10.4153/CMB-1971-028-8

[6] T.C. Clark and B. Falvai, N.D.R. Henderson and C.M. Mynhardt, Altitude of 4regular circulants, AKCE Int. J. Graphs Comb. 1 (2004) 149-166.

[7] E.J. Cockayne and G. Geldenhuys, P.J.P Grobler, C.M. Mynhardt and J.H. van Vuuren, The depression of a graph, Util. Math. 69 (2006) 143-160.

[8] E.J. Cockayne and C.M. Mynhardt, Altitude of $K_{3, n}$, J. Combin. Math. Combin. Comput. 52 (2005) 143-157.

[9] E.J. Cockayne and C.M. Mynhardt, A lower bound for the depression of trees, Australas. J. Combin. 35 (2006) 319-328.

[10] T. Dzido and H. Furmańczyk, Altitude of wheels and wheel-like graphs, Cent. Eur. J. Math. 8 (2010) 319-326. doi:10.2478/s11533-010-0017-4

[11] I. Gaber-Rosenblum and Y. Roditty, The depression of a graph and the diameter of its line graph, Discrete Math. 309 (2009) 1774-1778. doi:10.1016/j.disc.2008.03.002

[12] R.L. Graham and D.J. Kleitman, Increasing paths in edge ordered graphs, Period. Math. Hungar. 3 (1973) 141-148. doi:10.1007/BF02018469

[13] J. Katrenič and G. Semanišin, Finding monotone paths in edge-ordered graphs, Discrete Appl. Math. 158 (2010) 1624-1632.

doi:10.1016/j.dam.2010.05.018

[14] C.M. Mynhardt, Trees with depression three, Discrete Math. 308 (2008) 855-864. doi:10.1016/j.disc.2007.07.039

[15] C.M. Mynhardt, A.P. Burger and T.C. Clark, B. Falvai, and N.D.R. Henderson, Altitude of regular graphs with girth at least five, Discrete Math. 294 (2005) 241257. doi:10.1016/j.disc.2005.02.007

[16] C.M. Mynhardt and M. Schurch, A class of graphs with depression three, Discrete Math. 313 (2013) 1224-1232. doi:10.1016/j.disc.2012.05.011

[17] Y. Roditty, B. Shoham and R. Yuster, Monotone paths in edge-ordered sparse graphs, Discrete Math. 226 (2001) 411-417. doi:10.1016/S0012-365X(00)00174-6

[18] R. Yuster, Large monotone paths in graphs with bounded degree, Graphs Combin. 17 (2001) 579-587. doi:10.1007/s003730170031 
Received 5 November 2012

Revised 26 February 2013

Accepted 26 February 2013 